\title{
Metodologia de Ensino baseada em Avaliações Colaborativas e Rubricas para o Aprimoramento da Habilidade de Escrita de Relatórios Técnicos em Cursos de Graduação
}

\author{
Tiago de Oliveira ${ }^{1}$, Denise Stringhini ${ }^{1}$, José J. S. Craibas $^{1}$, Deborah G. M. Corrêa ${ }^{1}$ \\ ${ }^{1}$ Instituto de Ciência e Tecnologia - Universidade Federal de São Paulo (Unifesp) \\ Unidade Parque Tecnológico - CEP: 12231-280 - São José dos Campos - SP - Brasil \\ \{tiago.oliveira, dstringhini, jose.craibas, deborah.correa\}@unifesp.br
}

\begin{abstract}
This article presents a teaching methodology structured in a combination of online peer assessment and scoring rubric techniques with the objective of allowing students to follow and evaluate their peers work, attempting to find a more favorable educational environment focused on the development of written communication skills. The methodology was applied in an undergraduate course whose results are shown to be very promising by influencing the students to produce better technical reports as learning outcomes in contrast to tradicional methodology.

Resumo. Este artigo apresenta uma metodologia de ensino baseada em uma combinação das técnicas de avaliação colaborativa e rubrica com o objetivo de permitir que os alunos acompanhem e avaliem os trabalhos de seus pares buscando um ambiente educacional mais favorável ao desenvolvimento de habilidades de comunicação escrita. A metodologia foi aplicada em um curso de graduação cujos resultados obtidos mostram-se promissores ao ter influenciado os alunos na confecção de melhores relatórios técnicos como consequência do aprendizado se comparados a metodologia tradicional.
\end{abstract}

\section{Introdução}

A comunicação escrita é uma ferramenta fundamental em diversas situações para o sucesso profissional [França 2013]. Um profissional, de qualquer área, precisa conhecer bem seu idioma e suas normas de escrita para ser capaz de elaborar textos concisos e bem organizados que transmitam de forma clara objetivos, realização de projetos, pontos de vista, intenções, entre outras informações.

Devido a importância de se desenvolver a habilidade de escrita em alunos de cursos de graduação no Brasil, nos referenciais orientadores de currículos de diversos cursos de graduação tem-se incluído a necessidade de comunicação escrita nos perfis de egressos. Como exemplo, o MEC aprovou a resolução CNE/CES n. 11 em 2002, instituindo diversas diretrizes para todos os cursos de graduação em engenharia do país, onde pode-se destacar o inciso VIII do artigo 4 sobre competências e habilidades gerais de um engenheiro: comunicar-se eficientemente nas formas escrita, oral e gráfica. Outro exemplo que pode ser citado refere-se ao documento orientador de formação para cursos de graduação em Computação [Zorzo et al. 2017] publicado em 2017 pela Sociedade Brasileira de Computação (SBC) contendo a descrição do perfil de egresso para alunos de computação, destacando-se: ser capaz de se expressar verbalmente e na forma escrita; $e$ 
VII Congresso Brasileiro de Informática na Educação (CBIE 2018)

Anais do XXIX Simpósio Brasileiro de Informática na Educação (SBIE 2018)

de avaliar corretamente seus resultados e de terceiros. Saber transferir conhecimento e se manter atualizado.

Este artigo traz uma metodologia de ensino-aprendizagem por meio de avaliações colaborativas e o uso combinado de rubricas com a intenção de permitir ou estimular o desenvolvimento da habilidade de escrita nos alunos na confecção de relatórios técnicos, buscando-se uma alternativa mais eficaz em relação aos métodos tradicionais empregados nos currículos de cursos de graduação.

Na sequência, na seção 2, são introduzidos os conceitos de avaliação colaborativa e de rubricas como métodos de ensino-aprendizagem. Na seção 3, define-se a metodologia de ensino-aprendizagem mencionada anteriormente e, na seção 4, especifica-se o emprego dessa metodologia em uma disciplina de Laboratório de Arquitetura e Organização de Computadores de um curso de Engenharia de Computação. Na seção 5, apresentam-se os resultados de desempenho obtidos pelos alunos com a aplicação dessa metodologia, comparando-os com o desempenho de turmas submetidas a metodologia tradicional. Por fim, na seção 6, encontram-se as considerações finais sobre a metodologia aplicada e os trabalhos futuros a serem realizados.

\section{Fundamentação Teórica}

\subsection{Avaliação Colaborativa}

A avaliação colaborativa ou avaliação por pares [Tenório et al. 2016] é uma alternativa a avaliação tradicional, buscando unir a etapa de avaliação ao processo de ensinoaprendizagem do aluno e permitindo ao aluno o acompanhamento, a avaliação e a regulação da sua própria aprendizagem.

A avaliação colaborativa pode ser definida como um cenário em que alunos atuam na revisão de trabalhos de outros alunos de mesmo nível por meio de um acordo ou conjunto de critérios definidos pelo professor [Trahasch 2004] [Topping 2006]. Enquanto avaliados, seus trabalhos são revisados pelos colegas e, enquanto avaliadores, realizam retorno sobre os trabalhos de seus colegas. Todo esse processo pode possibilitar que os alunos pensem mais profundamente sobre determinado assunto, conduzindo-os a uma aprendizagem mais significativa [Trahasch 2004], onde professor e aluno podem refletir sobre os objetivos alcançados e adotar medidas para a superação de dificuldades em direção aos objetivos desejados. Além disso, quando um aluno avalia trabalhos desenvolvidos por seus colegas, indiretamente, o aluno está aprendendo a se autoavaliar e a ajustar o seu próprio trabalho em direção aos objetivos estabelecidos, desenvolvendo assim senso crítico e capacidade de análise e de reflexão sobre os objetivos desejados e alcançados. Assim, a avaliação passa a ser uma compilação de ações e intenções, centrada no aluno e não no professor.

A avaliação colaborativa tem sido aplicada com sucesso no ensino superior em áreas diversificadas, podendo-se citar, como exemplos, o trabalho publicado em [Wang et al. 2012] sobre o uso dessa prática de ensino na área de linguagens de programação em Computação, o trabalho publicado em [Ng 2014] para a formação de professores, o trabalho publicado em [Garousi 2010] numa disciplina relacionada a engenharia e os trabalhos nacionais publicados em [Silva and Moreira 2003] e [Ugulino et al. 2009] relacionados a aplicação da avaliação colaborativa em cursos de 
VII Congresso Brasileiro de Informática na Educação (CBIE 2018)

Anais do XXIX Simpósio Brasileiro de Informática na Educação (SBIE 2018)

graduação e pós-graduação em, respectivamente, disciplinas de computação e uma disciplina de Metodologia de Pesquisa Científica.

\subsection{Rubricas}

Uma definição comumente utilizada para rubrica refere-se a um documento que descreve a articulação das expectativas de uma determinada tarefa, listando-se os critérios ou quesitos que devem ser analisados e descrevendo-se os níveis de qualidade desejáveis em relação a cada um dos critérios discriminados [Reddy and Andrade 2010]. Essas tarefas podem corresponder aos mais variados tipos de objetos ou artefatos de aprendizagem, tais como, produtos ou protótipos, apresentações orais, mapas de conceitos, artigos, pôsteres e exames.

Esta prática de ensino pode ser incluída em dois contextos educacionais, quais sejam: na avaliação dos alunos e no processo de ensino-aprendizagem. No primeiro contexto, os artefatos produzidos pelos alunos podem ser avaliados e as rubricas utilizadas para aferição e estabelecimento de uma determinada nota ou conceito final relacionado a qualidade de cada um ou de um conjunto desses artefatos produzidos [Campbell 2005]. No segundo contexto, ao se disponibilizar antecipadamente critérios bem definidos e delineados da avaliação e os seus níveis de desempenho desejáveis, as rubricas podem ser utilizadas para facilitar a produção de retornos formativos (feedbacks) sobre o progresso dos alunos [Andrade 2000], além de servir como guia do processo de aprendizagem do próprio aluno, permitindo-o utilizar as rubricas como parâmetro no desenvolvimento, revisão e julgamento de seus trabalhos [Huba and Freed 2000].

Como exemplos de rubricas no ensino superior podem-se citar o desenvolvimento de um conjunto de ferramentas de software para facilitar a aplicação no ensino do quadro europeu de qualificações (European Qualifications Framework - EQF) relacionado ao processo de Bolonha [Florian-Gaviria et al. 2013]; a aplicação de rubricas para avaliação e para o aprendizado em cursos da área de economia [McGoldrick and Peterson 2013]; e os trabalhos nacionais sobre a avaliação de um Web fórum por meio de rubricas num curso de graduação em Ciências Biológicas [Ferreira and Silva 2010] e sobre a avaliação qualitativa de estudantes em cursos de computação e pedagogia [Lobato et al. 2009].

\section{Definição da Metodologia de Ensino}

O uso combinado da avaliação colaborativa e de rubricas pode ser estruturado para permitir o aprimoramento da habilidade de escrita em disciplinas que apresentem em seus conteúdos programáticos a execução de experimentos, o desenvolvimento de projetos ou a realização de tarefas/atividades que possam ser descritas por meio de relatórios técnicos.

A ideia é subdividir a disciplina em estágios ou pontos de checagem (PCs), sendo que no final de cada PC é realizada uma avaliação do progresso do aluno. Em cada um dos pontos de checagem, relatórios técnicos devem ser confeccionados pelos alunos da turma e um processo de avaliação colaborativa deverá ser realizado.

Quanto ao processo de avaliação colaborativa, relatórios técnicos confeccionados pelos alunos devem ser distribuídos entre seus pares para avaliação. A cada novo PC, novos relatórios técnicos são confeccionados e, por consequência, atribuídos aos alunos para avaliação. 
VII Congresso Brasileiro de Informática na Educação (CBIE 2018)

Anais do XXIX Simpósio Brasileiro de Informática na Educação (SBIE 2018)

Para nortear e balizar o processo de avaliação colaborativa realizado pelos alunos, um modelo de ficha de avaliação, apresentado na tabela 1, foi elaborado para o relatório técnico. Sendo assim, uma ficha de avaliação deve ser preenchida pelos alunos durante o processo de avaliação colaborativa, permitindo aos alunos conhecerem previamente os assuntos que deverão ser abordados em seus relatórios técnicos e como esses relatórios serão avaliados. Vale a pena observar que inclusões ou modificações no modelo de ficha de avaliação elaborado podem e devem ser realizadas para que se adequem aos objetivos esperados e as especificidades de cada PC definido.



Nesta tabela, observa-se que o modelo de ficha de avaliação elaborado estruturase em nove dimensões, quais sejam: cabeçalho, introdução, objetivos, fundamentação teórica, desenvolvimento do trabalho, resultados obtidos e discussão, considerações finais, referências bibliográficas e aspectos gerais. Para cada dimensão existe um conjunto de perguntas e uma seção final de comentários. Por sua vez, para cada pergunta existe uma escala ou estratificação de pontuação, devendo os alunos assinalar uma dentre as estratificações disponíveis para cada pergunta de acordo com a qualidade das respostas encontradas no relatório técnico que está em análise. Além disso, no final de cada dimensão, é de extrema importância que os alunos preencham a seção de comentários, descrevendo os pontos fortes e os pontos fracos da dimensão em análise, explicitando possíveis melhorias ou caminhos a serem percorridos.

A metodologia de ensino estrutura-se numa combinação de rubricas e avaliações 
VII Congresso Brasileiro de Informática na Educação (CBIE 2018)

Anais do XXIX Simpósio Brasileiro de Informática na Educação (SBIE 2018)

colaborativas com o intuito de (1) permitir aos alunos acompanharem os trabalhos que estão sendo desenvolvidos por outros alunos e observarem as diversas estratégias adotadas, os caminhos que estão sendo seguidos, as ferramentas que estão sendo utilizadas e as dificuldades que estão sendo encontradas pelos seus pares; (2) possibilitar que os alunos interajam entre si, propondo novos caminhos, alternativas e relatando seus pontos de vistas sobre os trabalhos que estão sendo desenvolvidos pelos seus pares, buscando-se, com isso, uma aprendizagem mais significativa e; (3) desenvolver nos alunos senso crítico e capacidade de auto-avaliação e auto-reflexão ao analisarem os trabalhos que estão sendo desenvolvidos pelos seus pares e, por consequência, seus próprios trabalhos.

\section{Aplicando a Metodologia de Ensino na Prática}

Na Universidade Federal de São Paulo - Unifesp, o ensino de arquitetura e organização de computadores está inserido num currículo de curso de Engenharia de Computação diferenciado, estruturado em uma abordagem prática e sistêmica que busca, além de integrar teoria e prática e também hardware e software, reduzir a visão fragmentada dos alunos de um sistema computacional complexo. Mais detalhes sobre essa estrutura curricular diferenciada da Unifesp podem ser encontrados no projeto pedagógico do curso ${ }^{1}$ e no artigo publicado em [Oliveira et al. 2017].

Dentro dessa estrutura encontra-se a disciplina de Laboratório de Arquitetura e Organização de Computadores, a qual ocorre em 4 horas semanais durante vinte semanas para turmas de 25 alunos, onde cada aluno deve desenvolver em linguagem de descrição de hardware Verilog sua própria plataforma de hardware, composta por processador, memória e sistema de entrada/saída utilizando um kit didático específico.

Para o cumprimento desses objetivos, a metodologia de ensino foi subdividida em quatro pontos de checagem (PCs), sendo eles: no primeiro ponto de checagem (PC1) os alunos são estimulados a pesquisar o funcionamento e arquitetura interna de vários processadores da literatura e a começarem a definir o conjunto de instruções (ISA) que será suportado pela sua plataforma de hardware; no segundo ponto de checagem (PC2) o aluno deve realizar um detalhamento maior do esboço da arquitetura interna e projetar em Verilog a unidade de processamento da plataforma de hardware; no terceiro ponto de checagem (PC3) o aluno deve realizar a especificação em Verilog da unidade de controle da plataforma de hardware proposta e, por fim, no quarto e último ponto de checagem (PC4) o aluno deve trabalhar na integração de todos os módulos produzidos, interligando a unidade de processamento e de controle da plataforma de hardware.

Em cada um dos quatro pontos de checagem, um relatório técnico deve ser confeccionado por aluno e um processo de avaliação colaborativa deverá ser realizado. Quanto ao processo de avaliação colaborativa, são atribuídos seis relatórios técnicos por aluno. A cada novo PC, atribuem-se relatórios técnicos de diferentes alunos, buscando-se evitar a repetição de alunos em relação aos pontos de checagem anteriores para um determinado avaliador. Sendo assim, no final da disciplina, um aluno terá analisado 24 relatórios técnicos de alunos diferentes, o que permite ao aluno ter tido contato com os projetos que estão sendo desenvolvidos por todos os alunos da turma, já que a disciplina possui no

\footnotetext{
${ }^{1} \mathrm{O}$ projeto pedagógico do curso de graduação em Engenharia de Computação da Unifesp foi aprovado em 2015 e pode ser encontrado pelo endereço eletrônico http://www.unifesp.br/campus/sjc/o-cursoengcom/projeto-pedagogico-do-curso.html, acessado em 03/09/2018.
} 
VII Congresso Brasileiro de Informática na Educação (CBIE 2018)

Anais do XXIX Simpósio Brasileiro de Informática na Educação (SBIE 2018)

máximo 25 alunos no total.

Por fim, o ambiente virtual de aprendizagem Moodle $^{2}$ foi utilizado para suportar a aplicação da metodologia de ensino, tendo sido organizado para permitir a realização, o mapeamento e a submissão das atividades propostas em cada ponto de checagem. $\mathrm{O}$ ambiente virtual Moodle foi escolhido por ser a plataforma institucional adotada na Unifesp, facilitando a configuração de contas, gerenciamento de conteúdo, agendamento e programação de atividades, distribuição de mensagens, aplicação das rubricas e realização da avaliação colaborativa. Além disso, os alunos já estão familiarizados com essa plataforma por a terem utilizado em outras unidades curriculares.

\section{Resultados Obtidos e Discussão}

Para analisar a qualidade dos relatórios técnicos produzidos pelos alunos por meio da avaliação colaborativa e rubricas utilizadas, como proposto na metodologia de ensino da seção 3, foi realizada uma comparação das notas dos alunos no último relatório produzido por eles durante quatro anos de oferecimento da disciplina de Laboratório de Arquitetura e Organização de Computadores (representados pelas turmas 01, 02, 03 e 04). Para todas as turmas, o último relatório de cada aluno foi entregue para um especialista da área que atribuiu uma nota de avaliação.

Para as turmas 03 e 04 foram aplicadas a metodologia de ensino proposta nesse artigo, enquanto que para as turmas 01 e 02 foi adotada uma metodologia de ensino tradicional: durante o semestre, após a ocorrência de aproximadamente $\frac{1}{3}, \frac{1}{2}, \frac{3}{4}$ e no final da unidade curricular, um relatório técnico era produzido por cada aluno. As correções desses relatórios, realizadas pelo professor responsável, eram entregues aos alunos contendo erros produzidos na redação dos textos dos relatórios, erros de especificação e implementação dos projetos e, quando necessário, instruções a serem realizadas para um realinhamento ou reposicionamento dos projetos para o correto desenvolvimento das plataformas de hardware.

Sendo assim, na figura 1(a), encontra-se a média das notas obtidas pelas turmas 01 , 02, 03 e 04 em relação ao último relatório técnico produzido pelos alunos da disciplina. No total, como tamanho das amostras, 17 relatórios técnicos foram entregues pela turma 01, enquanto 18 relatórios foram entregues em cada uma das demais turmas analisadas.

Para a turma 01, a média das notas obtidas no último relatório técnico elaborado pelos alunos foi de aproximadamente 7,86, para a turma 02 a média foi de aproximadamente 7,95 , para a turma 03 a média foi de aproximadamente 8,90 e para a turma 04 a média foi de aproximadamente 8,99. Portanto, com a aplicação da metodologia de ensino proposta, o desempenho dos alunos, representado pela média das notas obtidas no último relatório técnico produzido, é superior ao desempenho dos alunos que cursaram a disciplina na abordagem tradicional.

Uma informação estatística interessante pode ser relacionada a confiabilidade e a robustez das amostras obtidas referentes às notas dos relatórios técnicos produzidos pelos alunos das turmas 01, 02, 03 e 04 no final da disciplina, buscando-se comprovar estatis-

${ }^{2}$ O Moodle (Modular Object-Oriented Dynamic Learning Environment) é uma plataforma computacional de código aberto utilizado como apoio à aprendizagem do aluno. Mais detalhes sobre essa plataforma podem ser encontrados no endereço eletrônico https://moodle.org, acessado em 03/09/2018. 
VII Congresso Brasileiro de Informática na Educação (CBIE 2018)

Anais do XXIX Simpósio Brasileiro de Informática na Educação (SBIE 2018)

ticamente a melhora na habilidade de escrita dos alunos que foram submetidos a metodologia de ensino proposta. Devido a dimensão das amostras $(<30)$ e a utilização de escalas ordinais de medida (escala likert de 5 pontos), o teste estatístico não paramétrico Wilcoxon-Mann-Whitney, apresentado na figura 1(b), foi realizado com o auxílio da linguagem de programação $\mathrm{R}^{3}$ para verificar a equivalência entre o desempenho dos alunos na confecção dos relatórios técnicos. Nesta figura, encontram-se todas as combinações possíveis de comparação de desempenho entre as quatro turmas ofertadas.

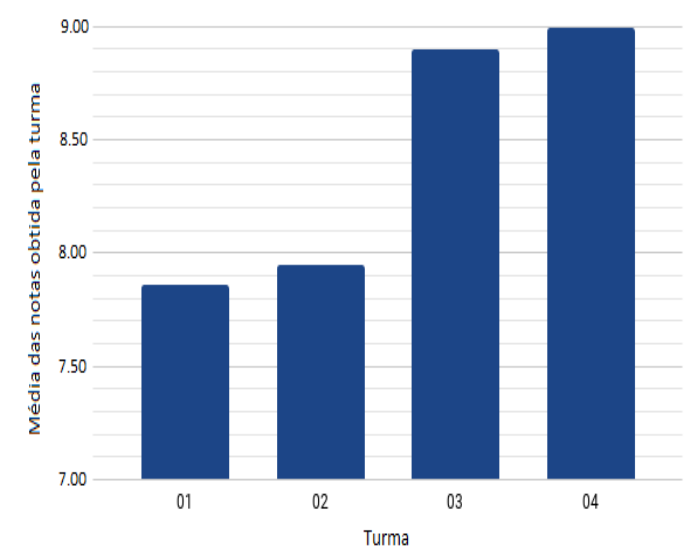

(a) Média obtida



(b) Teste de Wilcoxon-Mann-Whitney (equivalência das amostras)

Figura 1. Desempenho das turmas em relação ao último relatório técnico

Diante do gráfico apresentado na figura 1(b), a comparação estatística da turma 01 com a turma $03(01 / 03)$, da turma 01 com a turma $04(01 / 04)$, da turma 02 com a turma $03(02 / 03)$ e da turma 02 com a turma $04(02 / 04)$ resultou em p-valores menores do que o nível de significância de 0,05 delineado (linha tracejada no gráfico), rejeitandose, portanto, a hipótese nula de equivalência entre as turmas. Por sua vez, a comparação estatística entre as turmas 01 e $02(01 / 02)$ e entre as turmas 03 e $04(03 / 04)$ gerou pvalores maiores do que o nível de significância estabelecido $(0,05)$, aceitando-se, portanto, a hipótese nula e evidenciando a equivalência das amostras comparadas.

Particularmente esse resultado estatístico demonstra a confiabilidade e robustez das amostras, já que sinaliza claramente uma diferença entre os desempenhos dos alunos em relação às suas habilidades de escrita quando se comparam duas turmas submetidas a metodologias de ensino diferentes. Vale observar que, na figura 1(b), sempre que a comparação estatística ocorre entre uma turma 01 ou 02 (metodologia de ensino tradicional) com uma turma 03 ou 04 (metodologia de ensino proposta nesse artigo), aponta-se para uma diferença estatística significativa entre as turmas analisadas. Diferentemente do que ocorre ao se comparar a turma $01 \mathrm{com}$ a turma 02 (ambas turmas submetidas a metodologia de ensino tradicional) ou ao se comparar a turma 03 com a turma 04 (ambas turmas submetidas a metodologia de ensino proposta), pois nesses dois casos não se observam diferenças estatísticas significativas entre as turmas analisadas.

\footnotetext{
${ }^{3} \mathrm{O}$ software R utilizado é gratuito e implementa uma linguagem de programação específica além de um ambiente de desenvolvimento integrado para cálculos estatísticos e geração de gráficos. Para mais detalhes, consulte a página oficial do projeto R: https://www.r-project.org/, acessado em 12/06/2018.
} 
VII Congresso Brasileiro de Informática na Educação (CBIE 2018)

Anais do XXIX Simpósio Brasileiro de Informática na Educação (SBIE 2018)

Quanto a validade da análise sobre a melhora na habilidade de escrita dos alunos, o teste estatístico realizado anteriormente não é suficiente, fazendo-se necessário também verificar se o desempenho dos alunos das turmas 03 e 04, os quais foram submetidos a metodologia de ensino proposta, é estatisticamente superior ao desempenho dos alunos que cursaram a disciplina com a aplicação da metodologia tradicional, representados pelas turmas 01 e 02 .

Para tanto, levando em consideração que as amostras referentes às turmas $01 \mathrm{e}$ 02 não possuem diferença estatística significante para o nível de significância delineado, como pode ser observado na figura 1(b), e que, além disso, pertencem a aplicação de uma mesma metodologia de ensino, pode-se combiná-las para formar um único conjunto de dados contendo o desempenho dos alunos submetidos a aplicação da metodologia de ensino tradicional. Este conjunto de dados é, portanto, composto pela junção das amostras de desempenho da turma 01 e também da turma 02. De maneira análoga, podese combinar em um único conjunto de dados o desempenho obtido nas turmas 03 e 04, já que ambas as turmas foram submetidas a aplicação da metodologia de ensino proposta e não apresentam diferenças estatísticas significantes, de acordo com a figura 1(b).

Ao aplicar o teste estatístico por meio da linguagem $\mathrm{R}$ levando em consideração a hipótese alternativa de superioridade sobre o conjunto de dados contendo o desempenho combinado dos alunos das turmas 03 e 04 em comparação com o desempenho combinado dos alunos das turmas 01 e 02 , como mencionado no parágrafo anterior, obtém-se o pvalor de aproximadamente 0,001301 . Dessa forma, para o nível de significância delineado em 0,005, aceita-se a hipótese alternativa, comprovando-se a melhora na habilidade de escrita dos alunos que foram submetidos a metodologia de ensino proposta neste artigo.

\section{Considerações Finais}

Neste artigo apresentou-se uma metodologia de ensino estruturada em avaliações colaborativas para relatórios técnicos combinadas com a utilização de rubricas na definição de fichas de avaliação na tentativa de construir um ambiente didático que permita o aprimoramento da habilidade de escrita dos alunos. Como pôde ser constatado, essa abordagem de ensino-aprendizagem aplicada numa disciplina de um curso de Engenharia de Computação tem proporcionado a confecção de relatórios técnicos melhores se comparados com os relatórios produzidos numa abordagem de ensino tradicional, fomentando a interação entre os alunos, onde, por meio do preenchimento das fichas de avaliação, novos caminhos, alternativas, melhorias, ajustes e adequações são efetivados, estimulando-se, com isso, o senso crítico e a capacidade de auto-avaliação dos alunos ao analisarem os relatórios técnicos confeccionados pelos seus pares.

Como trabalho futuro, é interessante analisar não apenas a efetividade da metodologia de ensino sobre a habilidade de escrita técnica, mas também a percepção dos alunos sobre a adequação e eficiência da metodologia, utilizando-se, para isso, psicométricas definidas em instrumentos multidimensionais de avaliação do ensino. Com isso, será possível uma análise mais completa da metodologia abordando tanto o ponto de vista do professor responsável pela disciplina quanto o ponto de vista dos alunos sobre suas percepções da qualidade educacional empregada.

Além disso, buscar-se-á estender e ampliar a aplicação da metodologia de ensino abordada na seção 3 para outras disciplinas do curso de Engenharia de Computação e em 
VII Congresso Brasileiro de Informática na Educação (CBIE 2018)

Anais do XXIX Simpósio Brasileiro de Informática na Educação (SBIE 2018)

outros cursos da Unifesp. Assim, será possível observar com mais detalhes o comportamento da metodologia e sua influência em um conjunto de disciplinas.

Dentro deste contexto, vale ressaltar a capacidade de escalabilidade da metodologia de ensino-aprendizagem proposta neste artigo: com o processo de avaliação dos relatórios técnicos sendo compartilhado e distribuído com toda a turma, é possível aplicar a metodologia em disciplinas que possuem uma quantidade razoável de alunos. No entanto, se houver a intenção de que cada aluno tenha contato com todos os trabalhos que estão sendo desenvolvidos pelos demais alunos da turma, quanto maior o tamanho dessa turma, maior será a quantidade de relatórios técnicos que deverão ser atribuídos para cada avaliador. Dependendo do tamanho da turma, mesmo com o aumento na quantidade de relatórios técnicos por avaliador, pode-se tornar inviável garantir que cada aluno tenha contato com todos os trabalhos que estão sendo realizados pela turma.

No caso oposto, se a turma for muito pequena, pode não ser muito adequado cada relatório técnico ser avaliado por apenas um ou dois alunos, pois poderá ser gerada uma quantidade insuficiente de retornos formativos derivados das poucas fichas de avaliação preenchidas, levando a falhas na triangularização das informações disponibilizadas. Portanto, um estudo interessante que pode ser realizado refere-se à escalabilidade da metodologia de ensino proposta e a sua relação com a quantidade de fichas de avaliação atribuída por relatório técnico frente aos resultados de aprendizado de comunicação escrita apresentados pelos alunos.

Além das possibilidades de trabalhos futuros que foram descritas nos parágrafos anteriores, uma questão que merece um estudo mais aprofundado refere-se à inclusão de outras dimensões na ficha de avaliação para buscar o aprimoramento de algumas competências específicas. Por exemplo, a definição de uma dimensão sobre reflexão poderia fomentar, encorajar e intensificar ainda mais a auto-reflexão dos alunos. Seria interessante, portanto, a análise da inclusão dessa dimensão sobre o aprendizado dos alunos.

Por fim, a metodologia de ensino proposta também pode ser adaptada para ser aplicada em apresentações orais, buscando-se aprimorar não apenas a habilidade de comunicação escrita dos alunos, mas também a igualmente importante habilidade de comunicação verbal.

\section{Referências}

Andrade, H. (2000). Using rubrics to promote thinking and learning. Educational Leadership, 57(5):13-18.

Campbell, A. (2005). Application of ICT and rubrics to the assessment process where professional judgement is involved: the features of an e-marking tool. Assessment \& Evaluation in Higher Education, 30(5):529-537.

Ferreira, D. and Silva, A. (2010). Avaliação de um web fórum por meio de rubricas. Revista Meta: Avaliação, 2(4):87-127.

Florian-Gaviria, B., Glahn, C., and Fabregat Gesa, R. (2013). A Software Suite for Efficient Use of the European Qualifications Framework in Online and Blended Courses. IEEE Transactions on Learning Technologies, 6(3):283-296.

França, A. S. (2013). Comunicação Escrita Nas Empresas - Teorias e Práticas. Atlas. 
VII Congresso Brasileiro de Informática na Educação (CBIE 2018)

Anais do XXIX Simpósio Brasileiro de Informática na Educação (SBIE 2018)

Garousi, V. (2010). Applying Peer Reviews in Software Engineering Education: An Experiment and Lessons Learned. IEEE Transactions on Education, 53(2):182-193.

Huba, M. E. and Freed, J. E. (2000). Learner-centered Assessment on College Campuses: Shifting the Focus from Teaching to Learning. Allyn and Bacon, 8 edition.

Lobato, A. S., Brito, S. R., Souza, D. N. N., and Favero, E. L. (2009). Um sistema gerenciador de rubricas para apoiar a avaliação em ambientes de aprendizagem. In $X X$ Simpósio Brasileiro de Informática na Educação, Florianópolis/SC.

McGoldrick, K. and Peterson, B. (2013). Using rubrics in economics. International Review of Economics Education, 12:33-47.

$\mathrm{Ng}$, E. M. (2014). Using a mixed research method to evaluate the effectiveness of formative assessment in supporting student teachers' wiki authoring. Computers \& Education, 73:141-148.

Oliveira, T., Martins, L. E. G., Stringhini, D., Fazenda, A. L., and Cappabianco, F. A. M. (2017). O ensino e aprendizado de Arquitetura e Organização de Computadores num currículo de Engenharia de Computação estruturado em uma abordagem prática e sistêmica. International Journal of Computer Architecture Education (IJ$C A E), 6(1): 27-36$.

Reddy, Y. M. and Andrade, H. (2010). A review of rubric use in higher education. Assessment \& Evaluation in Higher Education, 35(4):435-448.

Silva, E. and Moreira, D. (2003). WebCoM: A Tool to Use Peer Review to Improve Student Interaction. Journal on Educational Resources in Computing, 3(1).

Tenório, T., Bittencourt, I. I., Isotani, S., and Silva, A. P. (2016). Does peer assessment in on-line learning environments work? a systematic review of the literature. Computers in Human Behavior, 64:94 - 107.

Topping, K. (2006). Self and Peer Assessment in School and University: Reliability, Validity and Utility. In Optimising New Modes of Assessment: In Search of Qualities and Standards, pages 55-87. Kluwer Academic Publishers, Dordrecht.

Trahasch, S. (2004). From peer assessment towards collaborative learning. In 34th Annual Frontiers in Education, 2004. FIE 2004., pages 774-778, Savannah, GA, USA. IEEE.

Ugulino, W., Marques, A. M., Pimentel, M., and Siqueira, S. W. (2009). Avaliação Colaborativa: um Estudo com a Ferramenta Moodle Workshop. In XX Simpósio Brasileiro de Informática na Educação, Florianópolis/SC.

Wang, Y., Li, H., Feng, Y., Jiang, Y., and Liu, Y. (2012). Assessment of programming language learning based on peer code review model: Implementation and experience report. Computers \& Education, 59(2):412 - 422.

Zorzo, A. F., Nunes, D., Matos, E. S., Steinmacher, I., Leite, J. C., Araujo, R., Correia, R. C. M., and Martins, S. (2017). Referenciais de Formação para os Cursos de Graduação em Computação. Sociedade Brasileira de Computação (SBC). 